\title{
Uso de álcool e drogas por estudantes de medicina da Unesp
}

\author{
Alcohol and drug use by Unesp medical students
}

Florence Kerr-Corrêa ${ }^{1}$, Arthur Guerra de Andrade ${ }^{2}$, Ana Zahira Bassit ${ }^{3}$, Neusa Maria Vilella Fonseca Boccuto ${ }^{4}$

Resumo Introdução: O objetivo deste trabalho foi analisar a prevalência do uso de drogas por estudantes da Faculdade de Medicina de Botucatu - Unesp, comparada com outras oito escolas médicas paulistas (uso na vida, nos últimos 12 meses e nos últimos 30 dias). A pesquisa foi realizada entre 1994 e 1995, com 5.227 estudantes do $1^{\circ}$ ao $6^{\circ}$ ano de graduação.

Material e método: Foi usado um questionário de auto-respostas, anônimo, incluindo o questionário da Organização Mundial da Saúde para levantamento de uso de drogas e álcool. Setenta e um por cento (3.725) dos alunos responderam ao mesmo, e destes, 421 eram de Botucatu.

Resultados: Não houve diferenças estatisticamente significantes entre escolas e, nos 30 dias anteriores ao preenchimento do questionário, a prevalência do uso de drogas para os estudantes de Botucatu foi a seguinte, com a variação entre outras escolas mostrada entre parênteses: álcool 50\% (42-50\%); tabaco 7\% (7-13\%); solventes 8\% (7-12\%); maconha 6\% (6-16\%); benzodiazepínicos (BZD) 3\% (2-9\%); cocaína 0,5\% (0,2-4\%); anfetaminas $1 \%(0-1 \%)$. Embora tenha se encontrado um uso crescente de todas as drogas do $1^{\circ}$ ao $6^{\circ}$ ano, e em especial os BZD, os estudantes não aprovam este uso. A análise de regressão logística indicou que o uso de álcool e drogas foi favorecido por: a) ser homem; b) perder aulas sem razão e referir ou ter muito tempo livre nos finais de semana; e c) ter uma atitude favorável em relação ao uso de álcool e drogas. Diferentemente de outras escolas, na Unesp não houve diferenças estatisticamente significantes de gênero em relação ao uso de tranqüilizantes. No entanto, as mulheres iniciam uso mais precocemente e o fazem mais freqüentemente. Também as mulheres já faziam uso de maconha antes de entrar para a faculdade (30\% mulheres X 10\% homens), o contrário ocorrendo com solventes (50\% homens X 2\% mulheres), sendo essas diferenças estatisticamente significantes.

Conclusões: Embora a pesquisa tenha focalizado o uso (não abuso ou dependência), os resultados sugerem a necessidade de as universidades estabelecerem uma política clara de orientação sobre uso de drogas e álcool para os estudantes, incluindo mudanças curriculares e programas de prevenção.

Descritores Uso de drogas e álcool; estudantes universitários; médicos; diferenças de gênero

Abstract Background: The objective of this paper was to analyse the prevalence of drug use by medical students of Botucatu Medical School, compared to other eight medical schools of São Paulo State (at lifetime, last 12 months, and last 30 days). Research was carried out in 1994 and 1995, with 5,227 students, from first to sixth year of graduation.

Method: Anonymous self-completed questionnaires were used, including the World Health Organization one, for students drug use survey. The completion rate was of $71 \%$ (3.725) and 421 were from Botucatu.

Results: There were no significant statistical differences among schools, and the last 30 day drug use rate showed the following results, for Botucatu and other schools (drug use range) respectively: alcohol in 50\% $(42-50 \%)$; tobacco in $7 \%(7-13 \%)$; inhalants in $8 \%$ (7-12\%); cannabis in 6\% (6-16\%); benzodiazepines (BZD) in $3 \%(2-9 \%)$; cocaine in $0,5 \%(0,2-4 \%)$; amphetamines in $1 \%(0-1 \%)$. Though there was an increased drug use from first to sixth year, especially BZD, most of the students did not approve of it. Risk factors for drug and alcohol use were: a) being men; b) truancy or excess free time on weekends; and c) approval of using alcohol

1. Professora Titular de Psiquiatria da Unesp.

2. Coordenador geral do Grupo Interdisciplinar de Estudos sobre Álcool e Drogas (Grea) do IPq HCFMUSP.

3. Psicólogo do Grupo Interdisciplinar de Estudos sobre Álcool e Drogas (Grea) do IPq HCFMUSP.

4. Psicóloga da UNESP. 
and drugs. Male and female students used equally BZD (contrary to other medical schools). However, women used them earlier and more frequently (female weekly X male monthly). Women used cannabis before entering school (female 30\% X male 19\%), being the contrary with solvent's use (statistically significant result).

Conclusions: Although the research focus was use (not abuse or dependence), the results suggest the necessity of campus drug and alcohol use policy to be developed and carried out for this population, with curricula modifications, and prevention program included.

Keywords Drug and alcohol use; college students; medical; students; gender differences

\section{Introdução}

O Brasil, a partir dos anos 80, e graças a inúmeros investigadores, bem como a uma política de incentivo à pesquisa científica sobre o tema (Ministério da Educação e col. 1990), é o país latino americano que tem gerado mais dados sobre dependência, bem como padrões de consumo de drogas e álcool em populações específicas, incluindo estudantes de $1^{\circ}$ e $2^{\circ}$ graus, ${ }^{1-4}$ estudantes universitários ${ }^{5-10}$ e graduandos de medicina., ${ }^{511-13}$

Por outro lado, a preocupação em detectar o uso e abuso de álcool e drogas, bem como atitudes em indivíduos com profissões ligadas à saúde é óbvia. Baseia-se na presunção de que tais usos e atitudes poderão interferir tanto na probabilidade de esses estudantes se tornarem médicos (no caso) dependentes ou com uso problemático de álcool ou drogas, como na habilidade dos mesmos de fazer o diagnóstico precoce, encaminhamento e/ou tratamento de pacientes dependentes. Baseia-se ainda no pressuposto de que o médico servirá de modelo para seus pacientes e outros profissionais de saúde que com ele convivem. Atualmente, a conduta de que o médico apenas indicaria o caminho a ser seguido, sem necessariamente o seguir, é pouco aceita.

Assim mesmo, faltam dados mais compreensivos de algumas populações, como por exemplo graduandos de medicina do interior do Estado, comparados aos da capital. Isso porque, faculdades de medicina do governo, pela dificuldade que oferecem no seu vestibular, têm suas vagas ocupadas em sua maioria por estudantes de fora da cidade ou região. Essa peculiaridade faz com que boa parte dos estudantes de medicina de faculdades interioranas não morem com seus pais e/ou parentes, fato já associado com maior uso de álcool e drogas. ${ }^{6,11}$

Aos fatos acima apontados, acrescem-se inúmeros acidentes, incluindo três mortes entre 1992 e 1993 envolvendo estudantes de medicina de Botucatu, nos quais o uso de drogas e álcool foi fator relevante.

$\mathrm{Na}$ ocasião, havendo preocupações semelhantes em outras escolas médicas, formou-se um consórcio de escolas médicas sob liderança de A.G. Andrade, que realizou um inquérito epidemiológico em nove faculdades de medicina que aceitaram participar do estudo. ${ }^{11} \mathrm{Na}$ Unesp, a responsável pela condução da pesquisa foi a Florence Kerr-Corrêa.

O presente trabalho teve por objetivos: 1) avaliar o uso de álcool e drogas entre os alunos da Faculdade de Medicina de Botucatu, comparados a graduandos de outras faculdades médicas do interior e da capital; 2) verificar se havia diferenças quantitativas e qualitativas em tal uso, com mais abuso nas cidades do interior, conforme algumas suspeitas; 3 ) verificar se estudantes de medicina usam mais ou menos álcool e drogas que outros estudantes universitários e 4) a partir dos resultados desse trabalho, analisar e dar subsídios para a implementação de medidas preventivas da faculdade de medicina da Unesp, nas demais participantes do consórcio e outras, eventualmente, que o desejarem.

\section{Sujeitos e métodos Sujeitos}

Fizeram parte do projeto todos os alunos de medicina, do primeiro ao sexto ano, matriculados nas nove seguintes faculdades paulistas no ano de 1994 ( $\mathrm{N}=5225)$ : Escola Paulista de Medicina (Unifesp); Faculdade de Ciências Médicas da Santa Casa de São Paulo; Faculdade de Ciências Médicas da Universidade Estadual de Campinas; Faculdade de Medicina do ABC; Faculdade de Medicina de Botucatu - Unesp; Faculdade de Medicina de Marília; Faculdade de Medicina de Santo Amaro; Faculdade de Medicina da Universidade de São Paulo; e Faculdade Regional de Medicina de São José do Rio Preto.

O N final foi de 3.725 , correspondentes a $71 \%$ dos alunos matriculados que responderam ao questionário. A Faculdade de Medicina de Botucatu tem 90 alunos matriculados por ano $(\mathrm{N}=540)$, dos quais 421 responderam ao questionário $(78 \%)$.

\section{Métodos}

Toda a coleta de dados foi realizada nos meses de outubro e novembro de 1994, através da aplicação de questionário de autopreenchimento proposto pela Organização Mundial da Saúde, ${ }^{14}$ a fim de levantar informações sobre o uso na vida, nos últimos 12 meses e nos últimos 30 dias, das seguintes substâncias: álcool, tabaco, maconha, alucinógenos, cocaína, anfetaminas, anticolinérgicos, solventes orgânicos, tranqüilizantes ansiolíticos, opiáceos, sedativos e barbitúricos. Além dessas informações, o questionário também apresentou questões sobre atitudes frente ao uso de substâncias psicoativas, atitudes frente a pacientes alcoolistas e farmacodependentes, e assuntos relacionados a drogas, qualidade de vida, lazer e dados sóciodemográficos.

Em todas as escolas médicas, os questionários foram aplicados durante as atividades acadêmicas (antes ou logo após as aulas) e com a anuência prévia dos professores que estavam 
ministrando estas aulas. Os alunos foram convidados a responder os questionários anonimamente, sendo que a ausência de respostas corresponde aos alunos que, ou não estavam presentes em sala de aula na hora da aplicação, ou se recusaram a responder o questionário.

A aplicação desses questionários foi padronizada e realizada por profissionais treinados especialmente para essa tarefa, em geral docentes que, antes do preenchimento do questionário, informavam os alunos quanto aos objetivos da pesquisa e forma pela qual os dados obtidos seriam tratados, reforçando o anonimato, a fim de conseguir maior fidedignidade dos relatos obtidos. Todo o processo de coleta de dados foi realizado durante um período de 30 dias, entre seu início e seu fim, sendo que cada aplicação do questionário nas salas de aula teve a duração média de vinte minutos.

A análise dos dados sobre a prevalência do uso de álcool e drogas foi realizada através do total geral de respostas afirmativas encontradas em cada escola, de acordo com o ano do curso médico e se a escola era do interior ou da capital. Os alunos de Botucatu foram comparados aos de outras escolas segundo a substância pesquisada quanto ao uso na vida, últimos 12 meses e últimos 30 dias. Realizou-se análise de regressão logística ${ }^{15}$ e verificou-se a associação entre as respostas ao uso de substâncias com o teste do "qui-quadrado" com correlação de Pearson, ${ }^{16}$ sendo considerado significância estatística quando $\mathrm{p}<0,05$.

\section{Resultados e discussão}

Não se verificou diferenças estatisticamente significantes entre alunos de Botucatu, outras faculdades do interior e da capital $^{11}$ quanto a dados sócioeconômicos gerais, de atitudes com relação ao uso de drogas, bem como ao uso propriamente dito das mesmas (na vida, nos últimos 12 meses, no último mês, na última semana).

Por isso, optou-se por apresentar o conjunto dos resultados, assinalando diferenças quando encontradas. A bem da verdade, a única diferença encontrada com relação a dados demográficos foi com relação à moradia: enquanto $77 \%$ dos alunos da capital residem com seus familiares, apenas $41 \%$ dos alunos do interior o fazem, sendo que $42 \%$ vivem em repúblicas. Assim mesmo, tal dado não influiu no uso maior de drogas como se poderia esperar, uma vez que no interior encontrou-se muitos alunos que moram sozinhos pela primeira vez, sem o (suposto) controle familiar. Foi diferente também do dado encontrado na USP. . $^{611}$

No uso de drogas em particular, assinala-se como foi o uso destas nos últimos 30 dias, comparados com as outras escolas, com suas variações mínimas e máximas ("range"). Além disso, questões freqüentemente perguntadas para cada droga em particular tiveram sua percentagem também anotada (por exemplo: $50 \%$ já experimentaram fumar tabaco e $30 \%$ fumaram no último mês, mas quantos usam diariamente?).

Com relação aos dados sóciodemográficos verificou-se, como o esperado, que em sua maioria os alunos de escolas médicas da capital ou do interior são de nível sócioeconômico alto: $40 \%$ têm renda superior a 20 salários mínimos/mês, o pai tem for- mação universitária em $63 \%$ e a mãe em $43 \%$ da amostra. Apesar de não haver diferenças estatisticamente significantes entre os alunos, $21 \%$ (capital) a $31 \%$ (interior) referiram que sobrava dinheiro que os pais Ihes davam todo mês. Provavelmente a diferença advém do fato de que o aluno que reside com os pais nem sempre tem mesada e, portanto, não tem como "fazer economia", "ter sobras". Assim mesmo, 9\% (interior) e $11 \%$ (capital), referiram ter que arranjar alguma complementação para viver. Nem sempre o aluno se sente à vontade para falar de renda familiar (37\% não responderam à questão). Mas é interessante notar que são, também, $9 \%$ aqueles que mencionam renda familiar menor que 10 salários mínimos.

A média da idade dos alunos de Botucatu foi de 21,7 anos (variação entre todas as escolas: 21,2 a 22,3 anos), predominando mulheres, de forma estatisticamente significante, nos grupos mais jovens. Quanto a estado civil e sexo, em todas as escolas predominaram os solteiros $(95 \%)$, sendo em média metade de cada sexo ( $47 \%$ mulheres e $53 \%$ de homens), uma tendência geral na busca atual da profissão médica no Brasil. Em Botucatu tem havido, nos últimos anos, maior número de mulheres que homens matriculados ( $1^{\circ}$ ano em 1995: $43 \%$ homens e $57 \%$ mulheres).

Os alunos como um todo consideraram seu desempenho escolar como bom, esperando obter realização profissional e financeira com a medicina. A maioria dos alunos tanto da capital como do interior tem apenas algumas horas livres nos finais de semana e de uma a quatro horas livres durante a semana. Quando faltam às aulas, $50 \%$ deles ficam em casa estudando, dormindo ou descansando. Quando têm oportunidade de lazer, as principais atividades por eles selecionadas foram, em ordem decrescente: assistir televisão, sair com amigos do mesmo sexo, ir ao cinema, sair com amigos do sexo aposto, ler jornais, ir a bares, praticar esportes e sair com namorado(a).

A maioria (95\%) dos alunos considera que o alcoolismo é um problema de saúde, mas menos de $25 \%$ recorreram a amigos, livros e aos pais quando tinham dúvidas sobre álcool e drogas. No entanto, quando o problema era sério, os alunos procuravam a mãe em primeiro lugar e o pai em segundo, seguido pelos amigos, irmãos e namorado(a). Raramente procuraram um professor.

Ao se analisar a distribuição dos alunos dos diferentes anos do curso médico, face à percepção sobre o tratamento de pacientes alcoolistas e farmacodependentes, verificou-se que mais de $50 \%$ dos alunos acreditam que esses pacientes não melhoram, sendo que esta percentagem aumentou no quarto, quinto e sexto anos. Os alunos também consideraram que esses pacientes são mais difíceis de tratar do que os outros, sendo que aproximadamente $80 \%$ dos alunos do sexto ano referiram que seu vínculo com pacientes alcoolistas e farmacodependentes era fraco ou ruim.

É interessante observar que a maioria dos alunos considerou que seu vínculo com outros pacientes era excelente ou bom, fato que poderia indicar, além do desconhecimento desses alunos em como tratar e lidar com alcoolistas e farmacodependentes, a baixa expectativa de melhora desses pacientes. No entanto, é relativamente fácil introduzir modificações. Trabalho anterior, em Botucatu, comparou a freqüên- 
cia de diagnóstico de alcoolismo antes e depois de modificações curriculares. Mesmo sem aumento de carga horária, a mudança do conteúdo programático na área de alcoolismo para a sua detecção precoce elevou de $8 \%$ para $18 \%$ a percentagem de diagnósticos realizados nos pedidos de interconsulta. ${ }^{17}$

Os alunos da capital e do interior, incluindo os da Unesp, desaprovaram tanto o uso experimental como o uso regular das seguintes substâncias, em ordem decrescente: cocaína, anfetamina, solventes, maconha e álcool, sendo que os alunos fizeram clara distinção entre o uso experimental de anfetaminas e solventes com o uso regular dessas substâncias. É interessante assinalar que a desaprovação se dá em ordem inversa da ordem de uso, com exceção dos solventes - como se lançaperfume, por exemplo, não fosse droga. Este achado corresponde, de fato, à tradição de muitas famílias brasileiras de utilizar solventes em carnavais e festas afins.

Dos alunos que experimentaram drogas, $60 \%$ não souberam explicar os motivos, $17 \%$ o fizeram por curiosidade e $9 \%$ por diversão ou prazer. Ao serem indagados sobre quem os introduziu ao uso experimental de droga, apontaram os amigos em primeiro lugar.

A análise do uso de álcool e drogas na vida, nos últimos 12 meses e nos últimos 30 dias, em relação ao total de respostas obtidas, tipo de substâncias e ano do curso médico, indicou a prevalência das seguintes substâncias: álcool, tabaco, solventes, maconha, tranquilizantes e cocaína. Nesta análise não se observou diferenças quanto ao padrão de uso dessas substâncias em relação ao uso na vida, nos últimos 12 meses e últimos 30 dias. Entretanto, o consumo das mesmas vai aumentando nos últimos anos do curso médico, o que diferencia esses resultados de outras pesquisas semelhantes. ${ }^{18-20}$ Impressiona o fato de que $2 / 3$ dos estudantes fumaram tabaco pela primeira vez após o início do curso médico, quando os trabalhos internacionais mostram que o contato com todas as drogas, com exceção de tranqüilizantes, começou muito antes do ingresso na faculdade. ${ }^{21,22}$ É possível, no entanto, que esse dado seja pouco confiável.

Quanto ao uso de tranqüilizantes em relação aos anos de curso médico, nota-se um consumo maior no sexto ano, tanto nos últimos 12 meses como nos últimos 30 dias. Ao contrário de outras drogas, o uso de tranqüilizantes se dá, na maioria das vezes, após o início da faculdade.

A tabela 1 sumariza os dados principais com relação ao uso de álcool e drogas pelos alunos de Botucatu, comparados aos das outras oito escolas de medicina do Estado de São Paulo. Os alunos da Unesp relataram usar álcool e drogas de forma comparável aos outros. Essa prevalência na Unesp e de outros estudantes de medicina em geral é menor que a encontrada entre outros universitários, incluindo médicos, para outras drogas que não o álcool ${ }^{23,5}$ e semelhante à encontrada para universitários americanos, de Brasília e São Paulo. ${ }^{8,13,21}$ Apesar desse uso ser experimental e esporádico, é preocupante pela importância da profissão no âmbito da saúde.

Impressiona, ainda, o fato de que solventes têm sido tão ou mais utilizados que a maconha pelos estudantes de medicina, dado diferente da literatura mundial e nacional para outros universitários. ${ }^{10,24}$ Uma possível explicação é a de que há facilidade de acesso gratuito a éter e clorofórmio dentro dos hospitais. Seria um provável fator para o achado de maior uso de solventes entre estudantes de medicina, maior até que maconha.

Houve tendência maior de uso de BZD (medicação de prescrição obrigatória) nos últimos anos de graduação. Podem se supor várias explicações: facilidade de obtenção de receitas e do próprio medicamento no hospital, estresse de final de curso com exame de residência a ser prestado e sobrecarga de plantões, com mudanças de turno freqüentes e rápidas, que atrapalham o ritmo do sono.

Os dados da literatura com relação ao uso de drogas por médicos são contraditórios. ${ }^{25}$ Havia a tendência, no passado, a considerá-los como população de risco ${ }^{24}$ Primeiramente pela facilidade de acesso aos medicamentos e, portanto, à automedicação. Em segundo lugar, pelo estresse inerente à profissão, que lida com a doença, a dor física e moral e a morte. Algumas especialidades seriam ainda mais susceptíveis (como anestesia e psiquiatria) pela facilidade de acesso às drogas.

Trabalhos mais recentes, porém, com metodologia mais apurada, não têm encontrado maior risco de uso de drogas por médicos. Estes as usariam de forma semelhante a outros profissionais de nível sócioeconômico semelhantes..$^{24,25}$ Em alguns países, outras profissões ofereciam maior risco de abuso de álcool, como seria o caso dos advogados americanos. ${ }^{25}$

Chamou ainda a atenção as poucas horas de lazer que têm. Nessas ocasiões, tendem a ficar assistindo TV ou a sair com amigos. Em Botucatu, particularmente, e nas outras escolas em geral, há poucas oportunidades de atividades culturais e/ou esportivas. O comum são idas a bares ou festinhas em repúbli-

Tabela 1 - Prevalência do uso de álcool e drogas por alunos de medicina de Botucatu (Unesp) e de outras oito escolas médicas: na vida, nos últimos $\mathbf{3 0}$ dias, uso semanal para álcool e diário para tabaco

\begin{tabular}{lcrrr} 
& \multicolumn{2}{c}{ Na vida } & \multicolumn{2}{c}{ No mês } \\
Droga & Unesp & outras & \multicolumn{2}{c}{ Unesp outras } \\
Álcool & $84 \%$ & $80-92 \%$ & $50 \%$ & $42-50 \%$ \\
Tabaco & $33 \%$ & $35-46 \%$ & $7 \%$ & $7-13 \%$ \\
Solventes & $30 \%$ & $25-38 \%$ & $8 \%$ & $7-12 \%$ \\
Maconha & $17 \%$ & $17-31 \%$ & $6 \%$ & $6-16 \%$ \\
Benzodiazepínicos & $14 \%$ & $8-16 \%$ & $3 \%$ & $2-9 \%$ \\
Cocaína & $3 \%$ & $3-7 \%$ & $0,5 \%$ & $0,2-4 \%$ \\
Anfetaminas & $6 \%$ & $4-16 \%$ & $1 \%$ & $0-1 \%$
\end{tabular}

\begin{tabular}{|c|c|}
\hline \multicolumn{2}{|c|}{ Na semana } \\
\hline Unesp & outras \\
\hline $23 \%$ & $23-31 \%$ \\
\hline - & - \\
\hline - & - \\
\hline - & - \\
\hline- & - \\
\hline - & - \\
\hline - & - \\
\hline
\end{tabular}

Diário Unesp outras

Obs: Opiáceos, alucinógenos e anticolinérgicos: sem uso significativo. 
cas (no interior), onde há uso freqüente de álcool e, às vezes, solventes e maconha. Há necessidade de uma análise estatística mais apurada desses dados, mas trabalho anterior de Mesquita e col. ${ }^{13}$ mostrou, entre outros fatores, que a freqüência constante a bares aumentava a probabilidade do uso de drogas em geral e de álcool em particular.

Não se encontraram diferenças estatisticamente significantes entre outras faculdades e a Unesp com relação ao padrão de uso de drogas para os dois gêneros, masculino e feminino. ${ }^{11}$ Os dados da Unesp com relação a gênero mostraram algumas diferenças no padrão de uso para os rapazes e moças, em relação a três categorias de drogas: a) tranquilizantes (BZD); b) solventes; e c) maconha.

a) embora não haja diferença estatisticamente significante entre o número de homens e mulheres que fazem uso de tranquilizantes (BZD) para os estudantes da Unesp, as mulheres iniciaram o uso mais precocemente (entre 17 e 20 anos) que seus colegas, que o fazem mais nos anos finais do curso e, portanto, com mais de 21 anos ( $p<0,005)$; elas também usam os BZD de forma mais intensa, semanalmente, enquanto os rapazes os usam mais esporadicamente, ou seja uma vez/mês ou menos - $(\mathrm{p}<0,05)$;

b) com relação aos solventes, enquanto $50 \%$ dos rapazes já os tinha usado antes de iniciar a faculdade, apenas $2 \%$ das mulheres o tinha feito $(\mathrm{p}<0,05)$;

c) já com a maconha, aconteceu o contrário: $71 \%$ dos rapazes e $29 \%$ das mulheres referiram já ter usado maconha. Porém, apenas $19 \%$ dos rapazes a tinha usado antes de iniciar a faculdade ( $<16$ anos), enquanto $30 \%$ delas já o tinha feito (X2 $=10,37 ; \mathrm{gl}=2 ; \mathrm{p}=0,0056)$, embora, no total, homens tenham referido em dobro seu uso nos últimos 30 dias com relação às mulheres $(23 \% \times 10 \%)$.

A análise logística mostrou que o uso de álcool e drogas pelos alunos de Botucatu, assim como das outras escolas, ${ }^{11}$ é favorecido por: 1) ser homem (exceto para BZD, quando ser mulher é um fator de risco); 2) perder aulas sem uma razão para isso, ou ter muito tempo nos finais de semana e 3) ter uma atitude favorável em relação ao uso de álcool e drogas.

Concluindo, há necessidade de melhor compreender os diferentes fatores envolvidos na resposta e na adaptação ao estresse inerentes ao curso de medicina para poder ajudar na prevenção do uso de drogas e álcool pelos futuros médicos. Uma política clara quanto ao uso de drogas e álcool pelos estudantes, informação científica, educação com treino de habilidades para melhor lidar com estresse, detecção precoce do uso de drogas têm se mostrado úteis na prevenção e têm sido adotados mundialmente. Não há, que se saiba, tal conduta em nenhuma escola médica brasileira. Normas e regras bem explicitadas, bem como

Tabela 2 - Uso de álcool e drogas, nos últimos 12 meses segundo o sexo, e início antes de entrar na faculdade por estudantes de medicina de Botucatu - Unesp

\begin{tabular}{|c|c|c|c|c|c|c|c|c|c|c|c|c|c|c|c|}
\hline & \multicolumn{4}{|c|}{ Homens } & \multicolumn{11}{|c|}{ Mulheres } \\
\hline & \multicolumn{2}{|c|}{ Não } & \multicolumn{2}{|c|}{ Sim } & \multirow[b]{2}{*}{$\%$} & \multirow[b]{2}{*}{ Total } & \multirow[b]{2}{*}{$\%$} & \multicolumn{2}{|c|}{ Não } & \multicolumn{2}{|c|}{ Sim } & \multirow[b]{2}{*}{$\left({ }^{*}\right)$} & \multirow[b]{2}{*}{$\%$} & \multirow[b]{2}{*}{ Total } & \\
\hline & $\mathrm{N}$ & $\%$ & $\mathrm{~N}$ & $\left(^{*}\right)$ & & & & $\mathrm{N}$ & $\%$ & & $\mathrm{~N}$ & & & & $\%$ \\
\hline Álcool & 30 & 14,6 & 176 & $(45)$ & 85,4 & 206 & 100,0 & 40 & 20,0 & 158 & $(61)$ & 80,0 & 198 & & 100,0 \\
\hline Tabaco & 171 & 82,6 & 36 & (12) & 17,4 & 203 & 100,0 & 169 & 85,4 & 29 & (12) & 14,6 & 198 & & 100,0 \\
\hline Solventes & 154 & 75,1 & 51 & (17) & 24,9 & 205 & 100,0 & 176 & 88,0 & 24 & (8) & 12,0 & 200 & & 100,0 \\
\hline Maconha* & 183 & 88,8 & 23 & (5) & 11,2 & 206 & 100,0 & 190 & 94,5 & 11 & (4) & 5,5 & 201 & & 100,0 \\
\hline Tranquilizantes** & 193 & 94,6 & 11 & (0) & 5,4 & 204 & 100,0 & 187 & 93,0 & 14 & (2) & 7,0 & 201 & & 100,0 \\
\hline Anfetaminas & 201 & 98,0 & 4 & (1) & 2,0 & 205 & 100,0 & 194 & 97,5 & 5 & (1) & 2,5 & 199 & & 100,0 \\
\hline Cocaína & 202 & 98,5 & 3 & (1) & 1,5 & 205 & 100,0 & 198 & 99,0 & 2 & (1) & 1,0 & 200 & & 100,0 \\
\hline
\end{tabular}

Tabela 3 - Associação entre idade de início do uso, sexo e uso de tranquilizantes nos últimos 12 meses para estudantes de medicina de Botucatu - UNESP - 1995.

\section{Tranquilizantes $=16$}

Sexo

$\begin{array}{llrlrr}\text { Homens * } & \text { Não } & 56 & 89 & 34 & 179 \\ & \text { Sim } & 1 & 11 & 11 & 23 \\ \text { Mulheres ** } & \text { Total } & 57 & 100 & 45 & 202 \\ & \text { Não } & 72 & 73 & 21 & 166 \\ & \text { Sim } & 7 & 18 & 8 & 33 \\ & \text { Total } & 79 & 91 & 29 & 199\end{array}$

* $X 2=12,86 ; g . l=2 ; p=0700161$

${ }^{*} X 2=6,62 ;$ g.l. $=2 ; p=003658$
Tabela 4 - Idade de início do uso de maconha (nos últimos 12 meses) em estudantes de medicina do sexo masculino da Faculdade de Medicina de Botucatu - Unesp

\begin{tabular}{lrrrr}
\multicolumn{2}{c}{ Uso no ano } & \multicolumn{2}{c}{ Idade (em anos) } & Total \\
maconha & $<\mathbf{1 6}$ & $\mathbf{1 7 - 2 0}$ & $>\mathbf{2 1}$ & \\
Não & 49 & 81 & 28 & 158 \\
Sim & 9 & 20 & 19 & 48 \\
Total & 58 & 101 & 47 & 206
\end{tabular}

$X 2=10,37 ; g . l .=2 ; p=0,0056$ 
o oferecimento (e mesmo encorajamento) de atividades recreativas e de relaxamento que não incluam substâncias alteradoras do psiquismo, um bom programa de professores-tutores (que seriam instruídos e treinados para detectar problemas dessa ordem) podem vir a melhorar a situação que, se não é pior que a dos demais universitários, está longe de ser boa.

A questão do uso de álcool e drogas entre alunos de medicina deve ser enfrentada como prioridade nas escolas médicas, que promoveriam programas de prevenção dirigidos especialmente a esta população, no qual deveria ser incluída a participação de professores, bem como também disciplina obrigatória (ou maior carga horária) sobre álcool e drogas nos cursos médicos. Esta pesquisa apresenta apenas uma parte da realidade das escolas médicas do Estado de São Paulo e considerase que pesquisas desta natureza devem ser ampliadas para outras escolas e outros estudantes universitários.

\section{Referências bibliográficas}

1. Carlini-Cotrin B, Carlini EA, Silva-Filho AR, Barbosa MTS. O uso de drogas psicotrópicas por estudantes de $1^{\circ}$ - e $2^{\circ}$. graus da rede estadual, em dez capitais brasileiras, 1987. In: Centro de Documentação do Ministério da Saúde. Brasília: DF, 1989. n.9-84. (Série C: Estudos e Projetos Consumo de drogas psicotrópicas no Brasil em 1987.)

2. Carlini EA, Carlini-Cotrin B, Silva-Filho AR, Barbosa MTS. Levantamento nacional sobre o uso de psicotrópico em estudantes de $1^{\circ}$ e $2^{\circ}$ graus, 1989. São Paulo: CEBRID/Escola Pauista Medicina (UNIFESP), 1990a.

3. Carlini-Cotrin B, Carlini EA, Silva-Filho AR, Barbosa MTS. 2. Levantamento nacional sobre o uso de psicotrópico por estudantes de $1^{\circ}$ e $2^{\circ}$ graus. São Paulo: CEBRID/Escola Paulista de Medicina (UNIFESP), 1990b.

4. Galduroz JCF, D'Almeida V, Carvalho V, Carlini EA. 3. Levantamento sobre uso de drogas entre estudantes de $1^{\circ}$ e $2^{\circ}$ graus em 10 capitais brasileiras (1993). São Paulo: CEBRID/ Escola Paulista de Medicina (UNIFESP), 1994.

5. Andrade AG, Bassit AZ, Mesquita AM, Fukushima JT, Gonçalves EL. Prevalência do uso de drogas entre alunos da Faculdade de Medicina da Universidade de São Paulo (1991-1993). Rev ABP-APAL 1995;17:41-6.

6. Andrade AG, Queiroz S, Villaboim RCM, César F, Alves MCGP, Bassit AZ, et al. Uso de álcool e drogas entre alunos de graduação da Universidade de São Paulo (1996). Rev ABP-APAL 1997;19:53-9.

7. Boskovitz EP, Cruz ETN, Chiaravalloti-Neto F, Moraes MS, Paiva-Netto JV, Avila LA, et al. Uso de drogas em universitários em São José do Rio Preto, São Paulo. Rev Psiquiatr Clin 1995;22:87-93.

8. Bücher RE, Totughi ML. Conocimiento y uso de drogas entre alunnos de Brasília. Acta Psiquiatr Psicol América Latina 1988;34:113-26.

9. Gorenstein C, DeLucia R, Gentil V. Uso de psicoestimulantes e energizantes por universitários. Rev Assoc Med Bras 1983;29:45-6.

10. Plotnik R, Azmus AD, Tannahauser M, Tannhauser SL. Utilização de psi- cotrópicos por estudantes universitários. Rev Pesq Med 1986;20:109-13.

11. Andrade AG, Bassit AZ, Kerr-Corrêa F, Tonhon AA, Boscovitz EP, Cabral $\mathrm{M}$, et al. Fatores de risco associados ao uso de álcool e drogas na vida, entre estudantes de medicina do Estado de São Paulo. Rev ABP-APAL 1997;19:11726.

12. Cruz AR, Cardoso JD. Avaliação do consumo e indução ao abuso de drogas entre alunos do curso de medicina. Ciência e Cultura 1988;40:935.

13. Mesquita AMC, Bucaretchi HA, Castel S, Andrade AG. Estudantes da Faculdade de Medicina da Universidade de São Paulo: uso de substâncias psicoativas em 1991. Rev ABP-APAL 1995;17:47-54.

14. Smart RG, Hughes PH, Johnstorn LD, Anumonye A, Krant V, Mora MEM, et al. A methodology for student drug-use surveys. Genebra, WHO Publication, 1980.

15. Kendal M. Multivariate analysis. Londres: Charles Griffin, 1980.

16. Siegel S. Estatística não paramédica. São Paulo: McGraw-Hill, 1977.

17. Kerr-Corrêa F, Smaira S1, Torres AR, Rossini R. É possível melhorar o diagnóstico de alcoolismo? Avaliação do ensino de psiquiatria através de interconsultas em enfermarias de um hospital universitário. Rev ABP-APAL $1989,2: 76-80$

18. Borini B, Oliveira CM, Martins MG, Guimarães RC. Conceitos, concepções etiológicas e atitudes de estudantes de medicina sobre o uso e abuso de álcool: correlações com os padrões de uso - Parte 2. J Bras Psiquiatr 1994;43:123-31.

19. Borini B, Oliveira CM, Martins MG, Guimarães RC. Médicos: uso e abuso de álcool. Conceitos e concepções etiológicas do alcoolismo - Parte 3. J Bras Psiquiatr 1994;43:213-19.

20. Borini B, Oliveira CM, Martins MG, Guimarães RC. Padrão de uso de bebidas alcoólicas de estudantes de medicina (Marília, São Paulo) - Parte 1. J Bras Psiquiatr 1994;43:93-103.

21. Baldwin-Jr DC, Hughes PH, Conard SE, Storr CL, Sheehan DV. Substance use among senior medical students. JAMA 1991;265:2074-78.

22. Schwartz RH, Lewis DC, Hoffmann NG, Kyriaki N. Cocaine and marijiana use by medical students before and during medical school. Arch Int Med 1990;150:883-86.

23. Magalhães MP, Barros RS, Silva MTA. Uso de drogas entre universitários: a experiência com maconha como fator delimitante. Rev ABP-APAL 1991;13: 97-104.

24. Hughes H, Brandenburg N, Baldwin-Jr DC, Storr CL, Williams KM, Anthony JC, et al. Prevalence of substance use among physicians. JAMA 1992;267:2333-39.

25. McAuliffe WE, Rohman M, Breer P, Wyslak G, Santangelo S, Magnurson E. Alcohol use and abuse in randon samples of physicians and medical students. Am J Public Health 1991;81:177-82.

\section{Correspondência}

Florence Kerr-Corrêa

Cx. Postal - 540

CEP: 18.618-970 - Botucatu, SP.

email: fcorrea@fmb.unesp.br 